CUBO A Mathematical Journal

Vol.15, $N^{0}$ 03, (45-50). October 2013

\title{
On centralizers of standard operator algebras with involution
}

\author{
Maja Fošner, Benjamin Marcen \\ Faculty of Logistics, \\ University of Maribor, \\ Mariborska cesta 73000 Celje Slovenia, \\ maja.fosner@fl.uni-mb.si, \\ benjamin.marcen@fl.uni-mb.si
}

\author{
NeJC ŠIROVNIK \\ Faculty of Natural Sciences and Mathematics, \\ University of Maribor, \\ Koroška cesta 1602000 Maribor Slovenia. \\ nejc.sirovnik@uni-mb.si
}

\begin{abstract}
The purpose of this paper is to prove the following result. Let $X$ be a complex Hilbert space, let $\mathcal{L}(X)$ be the algebra of all bounded linear operators on $X$ and let $\mathcal{A}(X) \subset$ $\mathcal{L}(\mathrm{X})$ be a standard operator algebra, which is closed under the adjoint operation. Let $\mathrm{T}: \mathcal{A}(\mathrm{X}) \rightarrow \mathcal{L}(\mathrm{X})$ be a linear mapping satisfying the relation $2 \mathrm{~T}\left(\mathrm{~A} \mathrm{~A}^{*} \mathrm{~A}\right)=\mathrm{T}(\mathrm{A}) \mathrm{A}^{*} \mathrm{~A}+$ $A A^{*} T(A)$ for all $A \in \mathcal{A}(X)$. In this case $T$ is of the form $T(A)=\lambda A$ for all $A \in \mathcal{A}(X)$, where $\lambda$ is some fixed complex number.
\end{abstract}

\section{RESUMEN}

El propósito de este artículo es probar el siguiente resultado. Sea $X$ un espacio de Hilbert complejo, sea $\mathcal{L}(X)$ el álgebra de todos los operadores lineales acotados sobre $X$ y sea $\mathcal{A}(X) \subset \mathcal{L}(X)$ la álgebra de operadores clásica, la cual es cerrada bajo la operación adjunto. Sea $\mathrm{T}: \mathcal{A}(\mathrm{X}) \rightarrow \mathcal{L}(\mathrm{X})$ una aplicación lineal satisfaciendo la relación $2 \mathrm{~T}\left(\mathrm{~A} \mathrm{~A}^{*} \mathrm{~A}\right)=\mathrm{T}(\mathrm{A}) \mathrm{A}^{*} \mathrm{~A}+\mathrm{A} \mathrm{A}^{*} \mathrm{~T}(\mathrm{~A})$ para todo $A \in \mathcal{A}(\mathrm{X})$. En este caso, $\mathrm{T}$ es de la forma $\mathrm{T}(\mathrm{A})=\lambda \mathrm{A}$ para todo $A \in \mathcal{A}(\mathrm{X})$, donde $\lambda$ es un número complejo fijo.

Keywords and Phrases: ring, ring with involution, prime ring, semiprime ring, Banach space, Hilbert space, standard operator algebra, $\mathrm{H}^{*}$-algebra, left (right) centralizer, two-sided centralizer.

2010 AMS Mathematics Subject Classification: 16N60, 46B99, 39B42. 
This research has been motivated by the work of Vukman, Kosi-Ulbl [5] and Zalar [13]. Throughout, $R$ will represent an associative ring with center $Z(R)$. Given an integer $n \geq 2$, a ring $R$ is said to be $n$-torsion free if for $x \in R, n x=0$ implies $x=0$. An additive mapping $x \mapsto x^{*}$ on a ring $\mathrm{R}$ is called involution if $(x y)^{*}=y^{*} x^{*}$ and $x^{* *}=x$ hold for all pairs $x, y \in R$. A ring equipped with an involution is called a ring with involution or ${ }^{*}$-ring. Recall that a ring $R$ is prime if for $a, b \in R, a R b=(0)$ implies that either $a=0$ or $b=0$, and is semiprime in case $a R a=(0)$ implies $a=0$. We denote by $Q_{r}$ and $C$ the Martindale right ring of quotients and the extended centroid of a semiprime ring $R$, respectively. For the explanation of $Q_{r}$ and $C$ we refer the reader to $[2]$.

An additive mapping $T: R \rightarrow R$ is called a left centralizer in case $T(x y)=T(x) y$ holds for all pairs $x, y \in R$. In case $R$ has the identity element, $T: R \rightarrow R$ is a left centralizer iff $T$ is of the form $T(x)=a x$ for all $x \in R$, where $a$ is some fixed element of $R$. For a semiprime ring $R$ all left centralizers are of the form $T(x)=q x$ for all $x \in R$, where $q \in Q_{r}$ is some fixed element (see Chapter 2 in [2]). An additive mapping $T: R \rightarrow R$ is called a left Jordan centralizer in case $\mathrm{T}\left(x^{2}\right)=\mathrm{T}(x) x$ holds for all $x \in R$. The definition of right centralizer and right Jordan centralizer should be self-explanatory. We call $T: R \rightarrow R$ a two-sided centralizer in case $T$ is both a left and a right centralizer. In case $T: R \rightarrow R$ is a two-sided centralizer, where $R$ is a semiprime ring with extended centroid $C$, then $T$ is of the form $T(x)=\lambda x$ for all $x \in R$, where $\lambda \in C$ is some fixed element (see Theorem 2.3.2 in [2]). Zalar [13] has proved that any left (right) Jordan centralizer on a semiprime ring is a left (right) centralizer.

Let us recall that a semisimple $H^{*}$-algebra is a complex semisimple Banach*-algebra whose norm is a Hilbert space norm such that $\left(x, y z^{*}\right)=(x z, y)=\left(z, x^{*} y\right)$ is fulfilled for all $x, y, z \in A$. For basic facts concerning $\mathrm{H}^{*}$-algebras we refer to [1]. Vukman [10] has proved that in case there exists an additive mapping $T: R \rightarrow R$, where $R$ is a 2-torsion free semiprime ring satisfying the relation $2 \mathrm{~T}\left(x^{2}\right)=\mathrm{T}(x) x+x \mathrm{~T}(x)$ for all $x \in R$, then $\mathrm{T}$ is a two-sided centralizer. Kosi-Ulbl and Vukman [9] have proved the following result. Let $A$ be a semisimple $H^{*}$-algebra and let $\mathrm{T}: \mathrm{A} \rightarrow \mathrm{A}$ be an additive mapping such that $2 \mathrm{~T}\left(x^{\mathrm{n}+1}\right)=\mathrm{T}(x) x^{\mathrm{n}}+x^{\mathrm{n}} \mathrm{T}(x)$ holds for all $x \in \mathrm{R}$ and some fixed integer $n \geq 1$. In this case $T$ is a two-sided centralizer. Recently, Benkovič, Eremita and Vukman [3] have considered the relation we have just mentioned above in prime rings with suitable characteristic restrictions. Kosi-Ulbl and Vukman [9] have proved that in case there exists an additive mapping $T: R \rightarrow R$, where $R$ is a 2-torsion free semiprime ${ }^{*}$-ring, satisfying the relation $\mathrm{T}\left(x x^{*}\right)=\mathrm{T}(x) x^{*}\left(\mathrm{~T}\left(x x^{*}\right)=x \mathrm{~T}\left(x^{*}\right)\right)$ for all $x \in \mathrm{R}$, then $\mathrm{T}$ is a left (right) centralizer. For results concerning centralizers on rings and algebras we refer to [4-13], where further references can be found.

Let $X$ be a real or complex Banach space and let $\mathcal{L}(X)$ and $\mathcal{F}(X)$ denote the algebra of all bounded linear operators on $X$ and the ideal of all finite rank operators in $\mathcal{L}(X)$, respectively. An algebra $\mathcal{A}(\mathrm{X}) \subset \mathcal{L}(\mathrm{X})$ is said to be standard in case $\mathcal{F}(\mathrm{X}) \subset \mathcal{A}(\mathrm{X})$. Let us point out that any standard operator algebra is prime, which is a consequence of a Hahn-Banach theorem. In case $X$ is a real or complex Hilbert space, we denote by $A^{*}$ the adjoint operator of $A \in \mathcal{L}(X)$. We denote 
by $X^{*}$ the dual space of a real or complex Banach space $X$.

Vukman and Kosi-Ulbl [5] have proved the following result.

Theorem 0.1. Let $\mathrm{R}$ be a 2-torsion free semiprime ring and let $\mathrm{T}: \mathrm{R} \rightarrow \mathrm{R}$ be an additive mapping. Suppose that

$$
2 \mathrm{~T}(x y x)=\mathrm{T}(\mathrm{x}) \mathrm{yx}+\mathrm{xy} \mathrm{T}(\mathrm{x})
$$

holds for all $\mathrm{x}, \mathrm{y} \in \mathrm{R}$. In this case $\mathrm{T}$ is a two-sided centralizer.

In case we have a ${ }^{*}$-ring, we obtain, after putting $y=x^{*}$ in the relation (1), the relation

$$
2 \mathrm{~T}\left(x x^{*} x\right)=\mathrm{T}(x) x^{*} x+x x^{*} \mathrm{~T}(x) .
$$

It is our aim in this paper to prove the following result, which is related to the above relation.

Theorem 0.2. Let $\mathrm{X}$ be a complex Hilbert space and let $\mathcal{A}(\mathrm{X})$ be a standard operator algebra, which is closed under the adjoint operation. Suppose $\mathrm{T}: \mathcal{A}(\mathrm{X}) \rightarrow \mathcal{L}(\mathrm{X})$ is a linear mapping satisfying the relation

$$
2 T\left(A A^{*} A\right)=T(A) A^{*} A+A A^{*} T(A)
$$

for all $\mathrm{A} \in \mathcal{A}(\mathrm{X})$. In this case $\mathrm{T}$ is of the form $\mathrm{T}(\mathrm{A})=\lambda \mathrm{A}$, where $\lambda$ is a fixed complex number.

Proof. Let us first consider the restriction of $\mathrm{T}$ on $\mathcal{F}(\mathrm{X})$. Let $A$ be from $\mathcal{F}(\mathrm{X})$ (in this case we have $A^{*} \in \mathcal{F}(X)$ ). Let $\mathrm{P} \in \mathcal{F}(X)$ be a self-adjoint projection with the property $A \mathrm{P}=\mathrm{PA}=\mathrm{A}$ (we also have $A^{*} \mathrm{P}=\mathrm{PA}^{*}=A^{*}$ ). Putting $\mathrm{P}$ for $\mathrm{A}$ in (2) we obtain

$$
2 \mathrm{~T}(\mathrm{P})=\mathrm{T}(\mathrm{P}) \mathrm{P}+\mathrm{PT}(\mathrm{P}) .
$$

Left multiplication by $\mathrm{P}$ in the above relation gives $\mathrm{PT}(\mathrm{P})=\mathrm{PT}(\mathrm{P}) \mathrm{P}$. Similarly, right multiplication by $\mathrm{P}$ in the above relation leads to $\mathrm{T}(\mathrm{P}) \mathrm{P}=\mathrm{PT}(\mathrm{P}) \mathrm{P}$. Therefore

$$
\mathrm{T}(\mathrm{P})=\mathrm{T}(\mathrm{P}) \mathrm{P}=\mathrm{PT}(\mathrm{P})=\mathrm{PT}(\mathrm{P}) \mathrm{P} .
$$

Putting $A+P$ for $A$ in the relation (2) we obtain

$$
\begin{aligned}
2 T\left(A^{2}\right) & +2 T\left(A A^{*}+A^{*} A\right)+4 T(A)+2 T\left(A^{*}\right)= \\
& =T(A)\left(A+A^{*}\right)+T(A) P+T(P) A^{*} A+T(P)\left(A+A^{*}\right)+ \\
& +\left(A+A^{*}\right) T(A)+P T(A)+A A^{*} T(P)+\left(A+A^{*}\right) T(P) .
\end{aligned}
$$

Putting $-A$ for $A$ in the above relation and comparing the relation so obtained with the above relation gives

$$
\begin{aligned}
& 2 T\left(A^{2}\right)+2 T\left(A A^{*}+A^{*} A\right)= \\
& \quad=T(A)\left(A+A^{*}\right)+T(P) A^{*} A+\left(A+A^{*}\right) T(A)+A A^{*} T(P)
\end{aligned}
$$


and

$$
\begin{aligned}
& 4 T(A)+2 T\left(A^{*}\right)= \\
& \quad=T(A) P+P T(A)+T(P)\left(A+A^{*}\right)+\left(A+A^{*}\right) T(P) .
\end{aligned}
$$

So far we have not used the assumption of the theorem that $X$ is a complex Hilbert space. Putting $i A$ for $A$ in the relations (4) and (5) and comparing the relations so obtained with the above relations, respectively, we obtain

$$
\begin{gathered}
2 T\left(A^{2}\right)=T(A) A+A T(A), \\
4 T(A)=T(A) P+P T(A)+T(P) A+A T(P) .
\end{gathered}
$$

Putting $A^{*}$ for $A$ in the relation (5) gives

$$
\begin{aligned}
4 T\left(A^{*}\right) & +2 T(A)= \\
& =T\left(A^{*}\right) P+P T\left(A^{*}\right)+T(P)\left(A+A^{*}\right)+\left(A+A^{*}\right) T(P) .
\end{aligned}
$$

Putting $i A$ for $A$ in the above relation and comparing the relation so obtained with the above relation leads to

$$
2 T(A)=T(P) A+A T(P) .
$$

Comparing the above relation and (7), we obtain

$$
2 T(A)=T(A) P+P T(A) .
$$

Right (left) multiplication by $\mathrm{P}$ in the above relation gives $\mathrm{T}(\mathrm{A}) \mathrm{P}=\mathrm{PT}(\mathrm{A}) \mathrm{P}$ and $\mathrm{PT}(\mathrm{A})=\mathrm{PT}(\mathrm{A}) \mathrm{P}$, respectively. Hence, $\mathrm{PT}(A)=\mathrm{T}(\mathrm{A}) \mathrm{P}$, which reduces the relation (8) to

$$
T(A)=T(A) P .
$$

From the above relation one can conclude that T maps $\mathcal{F}(X)$ into itself. We therefore have a linear mapping $T: \mathcal{F}(X) \rightarrow \mathcal{F}(X)$ satisfying the relation (6) for all $A \in \mathcal{F}(X)$. Since $\mathcal{F}(X)$ is prime, one can conclude, according to Theorem 1 in [10] that $T$ is a two-sided centralizer on $\mathcal{F}(X)$. We intend to prove that there exists an operator $C \in \mathcal{L}(X)$, such that

$$
T(A)=C A
$$

for all $A \in \mathcal{F}(X)$. For any fixed $x \in X$ and $f \in X^{*}$ we denote by $x \otimes f$ an operator from $\mathcal{F}(X)$ defined by $(x \otimes f) y=f(y) x, y \in X$. For any $A \in \mathcal{L}(X)$ we have $A(x \otimes f)=(A x) \otimes f$. Now let us choose such $f$ and $y$ that $f(y)=1$ and define $C x=T(x \otimes f) y$. Obviously, $C$ is linear and applying the fact that $\mathrm{T}$ is a left centralizer on $\mathcal{F}(\mathrm{X})$, we obtain

$$
(C A) x=C(A x)=T((A x) \otimes f) y=T(A(x \otimes f)) y=T(A)(x \otimes f) y=T(A) x
$$

for any $x \in X$. We therefore have $T(A)=C A$ for any $A \in \mathcal{F}(X)$. As $T$ is a right centralizer on $\mathcal{F}(X)$, we obtain $C(A B)=T(A B)=A T(B)=A C B$. We therefore have $[A, C] B=0$ for any 
$A, B \in \mathcal{F}(X)$, whence it follows that $[A, C]=0$ for any $A \in \mathcal{F}(X)$. Using closed graph theorem one can easily prove that $C$ is continuous. Since $C$ commutes with all operators from $\mathcal{F}(X)$, we can conclude that $C x=\lambda x$ holds for any $x \in X$ and some fixed complex number $\lambda$, which gives together with the relation (9) that $T$ is of the form

$$
\mathrm{T}(\mathrm{A})=\lambda \mathrm{A}
$$

for any $A \in \mathcal{F}(X)$ and some fixed complex number $\lambda$. It remains to prove that the relation (10) holds on $\mathcal{A}(\mathrm{X})$ as well. Let us introduce $\mathrm{T}_{1}: \mathcal{A}(\mathrm{X}) \rightarrow \mathcal{L}(\mathrm{X})$ by $\mathrm{T}_{1}(\mathrm{~A})=\lambda \mathrm{A}$ and consider $\mathrm{T}_{0}=\mathrm{T}-\mathrm{T}_{1}$. The mapping $T_{0}$ is, obviously, additive and satisfies the relation (2). Besides, $T_{0}$ vanishes on $\mathcal{F}(\mathrm{X})$. It is our aim to show that $T_{0}$ vanishes on $\mathcal{A}(\mathrm{X})$ as well. Let $A \in \mathcal{A}(\mathrm{X})$, let $\mathrm{P} \in \mathcal{F}(\mathrm{X})$ be a onedimensional self-adjoint projection and $S=A+P A P-(A P+P A)$. Such $S$ can also be written in the form $S=(I-P) A(I-P)$, where I denotes the identity operator on $X$. Since $S-A \in \mathcal{F}(X)$, we have $T_{0}(S)=T_{0}(A)$. It is easy to see that $S P=P S=0$. By the relation (2) we have

$$
\begin{aligned}
& \mathrm{T}_{0}(S) S^{*} S+S S^{*} T_{0}(S)= \\
& =2 T_{0}\left(S S^{*} S\right)= \\
& =2 T_{0}\left((S+P)(S+P)^{*}(S+P)\right)= \\
& =T_{0}(S+P)(S+P)^{*}(S+P)+(S+P)(S+P)^{*} T_{0}(S+P) \\
& =T_{0}(S) S^{*} S+T_{0}(S) P+S^{*} T_{0}(S)+P_{0}(S)
\end{aligned}
$$

We therefore have

$$
\mathrm{T}_{0}(\mathrm{~S}) \mathrm{P}+\mathrm{PT}_{0}(\mathrm{~S})=0 .
$$

Considering $T_{0}(S)=T_{0}(A)$ in the above relation, we obtain

$$
\mathrm{T}_{0}(A) \mathrm{P}+\mathrm{PT}_{0}(A)=0 .
$$

Multiplication from both sides by $\mathrm{P}$ in the above relation leads to

$$
\mathrm{PT}_{0}(\mathrm{~A}) \mathrm{P}=0 .
$$

Right multiplication by $\mathrm{P}$ in the relation (11) and considering the above relation gives

$$
\mathrm{T}_{0}(A) \mathrm{P}=0 \text {. }
$$

Since $\mathrm{P}$ is an arbitrary one-dimensional self-adjoint projection, it follows from the above relation that $T_{0}(A)=0$ for all $A \in \mathcal{A}(X)$, which completes the proof of the theorem.

We conclude the paper with the following conjecture.

Conjecture 0.3. Let $\mathrm{R}$ be a semiprime ${ }^{*}$-ring with suitable torsion restrictions and let $\mathrm{T}: \mathrm{R} \rightarrow \mathrm{R}$ be an additive mapping satisfying the relation

$$
2 \mathrm{~T}\left(x x^{*} x\right)=\mathrm{T}(x) x^{*} x+x x^{*} \mathrm{~T}(x)
$$

for all $x \in \mathrm{R}$. In this case $\mathrm{T}$ is a two-sided centralizer. 


\section{References}

[1] W. Ambrose: Structure theorems for a special class of Banach algebras, Trans. Amer. Math. Soc. 57 (1945), 364-386.

[2] K. I. Beidar, W. S. Martindale 3rd, A. V. Mikhalev: Rings with generalized identities, Marcel Dekker, Inc., New York, (1996).

[3] D. Benkovič, D. Eremita, J. Vukman: A characterization of the centroid of a prime ring, Studia Sci. Math. Hungar. 45 (3) (2008), 379-394.

[4] I. Kosi-Ulbl, J. Vukman: An equation related to centralizers in semiprime rings, Glas. Mat. 38 (58) (2003), 253-261.

[5] I. Kosi-Ulbl, J. Vukman: On centralizers of semiprime rings, Aequationes Math. 66 (2003), 277-283.

[6] I. Kosi-Ulbl, J. Vukman: On certain equations satisfied by centralizers in rings, Internat. Math. J. 5 (2004), 437-456.

[7] I. Kosi-Ulbl, J. Vukman: Centralizers on rings and algebras, Bull. Austral. Math. Soc. 71 (2005), 225-234.

[8] I. Kosi-Ulbl, J. Vukman: A remark on a paper of L. Molnár, Publ. Math. Debrecen. 67 (2005), 419-421.

[9] I. Kosi-Ulbl, J. Vukman: On centralizers of standard operator algebras and semisimple $\mathrm{H}^{*}$ algebras, Acta Math. Hungar. 110 (3) (2006), 217-223.

[10] J. Vukman: An identity related to centralizers in semiprime rings, Comment. Math. Univ. Carol. 40 (1999), 447-456.

[11] J. Vukman: Centralizers of semiprime rings, Comment. Math. Univ. Carol. 42 (2001), 237245.

[12] J. Vukman: Identities related to derivations and centralizers on standard operator algebras, Taiwan. J. Math. Vol. 11 (2007), 255-265.

[13] B. Zalar: On centralizers of semiprime rings, Comment. Math. Univ. Carol. 32 (1991), 609614. 